\title{
A new retuning approach for DoA reference tracking improvement
}

\author{
Filipa Nunes Nogueira ${ }^{*}$ Teresa Mendonça ${ }^{* *}$ Paula Rocha ${ }^{* * *}$ \\ * Faculdade de Engenharia da Universidade do Porto, Porto, Portugal \\ CIDMA - Center for Research and Development in Mathematics and \\ Applications, Universidade de Aveiro, Aveiro, Portugal, (e-mail: \\ dma09030@fe.up.pt) \\ ** Faculdade de Ciências da Universidade do Porto, Porto, Portugal \\ CIDMA - Center for Research and Development in Mathematics and \\ Applications, Universidade de Aveiro, Aveiro, Portugal, (e-mail: \\ tmendo@fc.up.pt) \\ *** Faculdade de Engenharia da Universidade do Porto, Porto, \\ Portugal \\ CIDMA - Center for Research and Development in Mathematics and \\ Applications, Universidade de Aveiro, Aveiro, Portugal, (e-mail: \\ mprocha@fe.up.pt)
}

\begin{abstract}
In this paper, a retuning strategy for a controller is proposed in order to improve the reference BIS tracking in patients, by means of simultaneous administration of propofol and of remifentanil, in the presence of model uncertainties. This strategy proves to be useful as is shown by simulations.
\end{abstract}

Keywords: Automatic control; DoA; Anesthesia.

\section{INTRODUCTION}

One of the most important concerns of the anesthetists during surgery is the administration of the right combination of drugs in order to achieve a desired depth of anesthesia (DoA). The DoA consists of two components, the hypnosis and the analgesia. The study presented in this paper concerns the case where hypnosis and analgesia are achieved, respectively, by the administration of propofol and of remifentanil. Moreover, following several studies (Tirén et al. [2005], Grindstaff and Tobias [2004], Ekman et al. [2004], Wodey et al. [2005], Whyte and Booker [2003]) and clinical practice, the level of DoA is measured by means of the bispectral index (BIS). This index is a single dimensionless number, which is computed from the electroencephalogram (EEG) and ranges from 0 (equivalent to EEG silence) to 100 (equivalent to fully awake and alert). According to medical practice a BIS value between 40 and 60 corresponds to an appropriate DoA level for general anesthesia.

After an adequate model for the BIS response of a patient to the administration of propofol and remifentanil is identified, the desired BIS value may be tracked by the automatic controller developed in Nogueira et al. [2014] (see also Nogueira et al. [2012]). However, in the presence of

\footnotetext{
* This work was supported by Portuguese funds through the CIDMA - Center for Research and Development in Mathematics and Applications, and the Portuguese Foundation for Science and Technology ("FCT-Fundação para a Ciência e a Tecnologia"), within project PEst-OE/MAT/UI4106/2014. Filipa Nogueira also acknowledges the support of FCT - Portugal through the grant SFRH/BD/48314/2008.
}

uncertainties, due to errors in modeling and identification procedures, the BIS obtained may be different from the desired one. In this work, we present a controller retuning strategy in order to overcome this problem. The proposed retuning strategy improves the BIS reference tracking as illustrated by simulations.

\section{MODEL DESCRIPTION}

The patient BIS response to the administration of propofol and of remifentanil is commonly modeled as a high order pharmacokinetic/pharmacodynamic (PK/PD) Wiener model, which is constituted by a dynamic linear part based on the relevant patient characteristics (see Bailey and Haddad [2005], Schnider et al. [1998], Marsh et al. [1991], Minto et al. [1997]) and by a nonlinear static part (see Minto et al. [2000]). However, a new Wiener model (parameter parsimonious Wiener model) with a reduced number of parameters describing the joint effect of propofol and of remifentanil has been introduced in Silva et al. [2010].

In the parameter parsimonious model (PPM) the relation between the propofol and remifentanil dosages and the corresponding effect concentrations $\left(c_{e}^{p}\right.$ and $\left.c_{e}^{r}\right)$ are modeled by the transfer functions: 


$$
\begin{aligned}
& H^{p}(s)=\frac{k_{1} k_{2} k_{3} \alpha^{3}}{\left(k_{1} \alpha+s\right)\left(k_{2} \alpha+s\right)\left(k_{3} \alpha+s\right)} u^{p}(s), \\
& H^{r}(s)=\frac{l_{1} l_{2} l_{3} \eta^{3}}{\left(l_{1} \eta+s\right)\left(l_{2} \eta+s\right)\left(l_{3} \eta+s\right)} u^{r}(s),
\end{aligned}
$$

respectively, where $\alpha$ and $\eta$ are patient dependent parameters, without any physiological meaning. The corresponding BIS level, $z(t)$, usually given by the generalized Hill equation Minto et al. [2000], is approximated in Silva et al. [2010] by the nonlinear equation:

$$
z(t)=\frac{z_{0}}{1+(U(t))^{\gamma}}
$$

where $U(t)=\mu U^{p}+U^{r}$, and $\mu$ and $\gamma$ are patient dependent parameters, without any physiological meaning, $z_{0}$ is the BIS level at zero concentration, and $U^{p}$ and $U^{r}$ respectively denote the potencies of propofol and remifentanil, which are obtained by normalizing the effect concentrations with respect to the concentrations that produce half the maximal effect when the drug acts isolated (denoted by $E C_{50}^{p}$ and $E C_{50}^{r}$, respectively), i.e.:

$$
U^{p}=\frac{c_{e}^{p}}{E C_{50}^{p}} \text { and } U^{r}=\frac{c_{e}^{r}}{E C_{50}^{r}}
$$

The parameters $E C_{50}^{p}$ and $E C_{50}^{r}$ are taken to be fixed, namely $E C_{50}^{p}=10 \mathrm{mg} / \mathrm{ml}$ and $E C_{50}^{r}=0.01 \mathrm{mg} / \mathrm{ml}$. These values were obtained in the work developed in Mendonça et al. [2012], to which we refer for further explanation.

\section{DEPTH OF ANESTHESIA CONTROLLER}

In the work developed in Nogueira et al. [2014] (see also Nogueira et al. [2012]), a nonlinear controller was presented in order to track a desired reference value for the BIS level, by means of simultaneous administration of propofol and of remifentanil. This controller results from a combination of a linear control law with a positivity constraint for the drug doses and is based on the state space realizations $\Sigma^{p}=\left(A^{p}, B^{p}, C^{p}\right)$ of the transfer function $H^{p}(s)$ and $\Sigma^{r}=\left(A^{r}, B^{r}, C^{r}\right)$ of $H^{r}(s)$. The matrices of such realizations are as follows:

$$
\begin{aligned}
& A^{p}=\left[\begin{array}{ccc}
-10 \alpha & 0 & 0 \\
9 \alpha & -9 \alpha & 0 \\
0 & \alpha & -\alpha
\end{array}\right], \\
& B^{p}=\left[\begin{array}{c}
10 \alpha \\
0 \\
0
\end{array}\right], \\
& C^{p}=\left[\begin{array}{lll}
0 & 0 & 1
\end{array}\right], \\
& A^{r}=\left[\begin{array}{ccc}
-3 \eta & 0 & 0 \\
2 \eta & -2 \eta & 0 \\
0 & \eta & -\eta
\end{array}\right], \\
& B^{r}=\left[\begin{array}{c}
3 \eta \\
0 \\
0
\end{array}\right], \\
& C^{r}=\left[\begin{array}{lll}
0 & 0 & 1
\end{array}\right] .
\end{aligned}
$$

Further, the states of $\Sigma^{p}$ and of $\Sigma^{r}$ are respectively denoted by $x^{p}$ and $x^{r}$.

The controller is then defined by:

$$
u(t)=\left[\begin{array}{l}
u^{p}(t) \\
u^{r}(t)
\end{array}\right]=\left[\begin{array}{l}
\max \left(0, \tilde{u}^{p}(t)\right. \\
\max \left(0, \tilde{u}^{r}(t)\right.
\end{array}\right],
$$

where $u^{p}$ is the input of propofol and $u^{r}$ is the input of remifentanil, with:

$$
\left[\begin{array}{l}
\tilde{u}^{p} \\
\tilde{u}^{r}
\end{array}\right]=E\left(-K A x+\lambda\left(M^{*}-K x\right)\right),
$$

where

$$
\begin{aligned}
& x=\left[\begin{array}{l}
x^{p} \\
x^{r}
\end{array}\right], \\
& A=\left[\begin{array}{cc}
A^{p} & 0 \\
0 & A^{r}
\end{array}\right] \\
& E=\left[\begin{array}{l}
\rho \\
1
\end{array}\right] \frac{1}{\alpha \rho+300 \eta}, \\
& M^{*}=\frac{3(0.1 \rho+100)}{0.1 \mu \rho+100}\left(\frac{z_{0}}{z^{*}}-1\right)^{\frac{1}{\gamma}}, \\
& K=\left[\begin{array}{llllll}
0.1 & 0.1 & 0.1 & 100 & 100 & 100
\end{array}\right],
\end{aligned}
$$

$z^{*}$ is the desired BIS level, and $\lambda$ and $\rho$ are positive design parameters that do not affect the tracked reference value and can be chosen according to clinical criteria. The parameter $\lambda$ influences the convergence speed to the desired reference value and the parameter $\rho$ can be interpreted as the proportion between the doses of propofol and remifentanil. 
For more details about this controller and its tracking properties, the reader is referred to Nogueira et al. [2014] or to Nogueira et al. [2012].

\section{RETUNING STRATEGY FOR DOA CONTROL}

In this section a strategy is presented to retune the controller described in Section 3 in order to overcome the problems raised by uncertainty in the BIS patient modeling process. The idea is to change the reference value for the parameter $M^{*}$ in the control law (7) according to the response of the patient when the controller is applied. For this purpose, we assume that the uncertainties in the model only occur in its linear part and in the parameter $\mu$, the parameter $\gamma$ of the Hill equation (3) is considered to be known. A simple procedure to identify the parameter $\gamma$ can be found in the work developed in Nogueira et al. [2013].

In the sequel, we denote by $M_{\text {model }}^{r e f}$ the target value used in the control law applied to the PPM used to describe a patient in order to achieve a certain reference value for the BIS, $U_{\text {model }}(t)$ denoted the theoretical potency $U(t)$ obtained for the PPM and $U_{\text {patient }}(t)$ denotes the potency $U(t)$ obtained for a real patient.

It follows from what has been presented in Nogueira et al. [2014], and summarized in the previous section, that when, in the controller (7), the parameter $M^{*}$ is set to:

$$
M^{*}=M_{\text {model }}^{\text {ref }}=\frac{3(0.1 \rho+100)}{0.1 \mu \rho+100}\left(\frac{z_{0}}{z^{*}}-1\right)^{\frac{1}{\gamma}},
$$

the desired BIS value $z^{*}$ is achieved to the corresponding PPM. This means that the potency $U_{\text {model }}(t)$ converges to a value

$$
\begin{aligned}
U^{*} & =\sqrt[\gamma]{\frac{z_{0}}{z^{*}}-1} \\
& =\tau M_{\text {model }}^{\text {ref }}
\end{aligned}
$$

with $\tau=\frac{0.1 \mu \rho+100}{3(0.1 \rho+100)}$.

However, when the controller is tuned with the parameter $M^{*}=M_{\text {model }}^{r e f}$ as in (13), the BIS level obtained in a real patient will be equal to a certain value $\bar{z}$ instead of the desired one, $z^{*}$. This means that $U_{\text {patient }}(t)$ converges to a value

$$
\bar{U}=\sqrt[\gamma]{\frac{z_{0}}{\bar{z}-1}}
$$

The retuning strategy presented here is based on the assumption that the patient is correctly modeled by a PPM. Moreover it is assumed that the misfit in the achieved BIS values is due to the fact that the actual patient PPM differs from the initially considered one. In this case, the same type of relationship exists as for the original PPM between the steady state value of $U(t)$ and the value of $M^{*}$ taken in the control law (7). I.e., setting $M^{*}=\bar{M}$ and applying the corresponding control law to the patient yields a steady state value of $U(t)$

$$
\bar{U}=\bar{\tau} \bar{M}
$$

for an adequate value of $\bar{\tau}$. Since $\bar{M}$ is known, the value of $\bar{\tau}$ can be computed by letting the system settle down and computing $\bar{U}$. In particular, if ones observers that setting $M^{*}=M_{\text {mod }}^{r e f}$ yields a state $U^{* *}\left(\neq U^{*}\right)$ for $U(t)$ one can conclude that

$$
\bar{\tau}=\frac{U^{* *}}{M_{m o d}^{r e f}} .
$$

This means that, in order to track the BIS reference value $z^{*}$, or equivalently in order to achieve the steady state value $U^{*}$ for $U(t)$, the adequate value for $M^{*}$ in $(7)$ should be given by:

$$
\begin{aligned}
M^{*} & =\frac{U^{*}}{\bar{\tau}} \Leftrightarrow \\
M^{*} & =\frac{U^{*}}{U * *} M_{m o d}^{r e f} .
\end{aligned}
$$

Therefore an improvement of the reference tracking performance can be obtained by means of the following strategy:

Given a PPM for a patient:

(1) Compute the value $M^{*}=M_{m o d}^{r e f}$ as in (11) according to the PPM parameters.

(2) Compute the theoretical steady state value $U^{*}$ of $U(t)$ according to equation (14).

(3) Apply the corresponding control law (7) to deliver the adequate doses of propofol and remifentanil.

(4) Observe the patient response, read out the BIS steady state value $z^{* *}$ and compute the corresponding steady state value $U^{* *}$ of the combined potency $U(t)$.

(5) Retune the value of $M^{*}$ in (7) according to the equation (20).

\section{SIMULATIONS}

In this section, the performance of the retuning strategy for the improvement of the performance of the controller (7) is illustrated by simulations. For this purpose, the control law is applied to a simulated patient that was set up based on the data of a real patient, a woman, with 68 years of age, a height of $158 \mathrm{~cm}$, and $113 \mathrm{Kg}$ who was subject to general anesthesia under propofol and remifentanil administration during a breast surgery. The DoA was monitored by the BIS and was manually controlled around clinically accepted values by the anesthetist. Alaris GH pumps were used for both propofol and remifentanil. Infusion rates, BIS values and other physiological variables were acquired every five seconds (Mendonça et al. [2012]).

For this patient, a PK/PD Wiener model (other than the PPM) was obtained as follows. The linear part was modeled according to Marsh et al. [1991], Minto et al. [1997], and Schnider et al. [1998] based on the relevant patient characteristics. The corresponding model is summarized in equation (1) of Ionescu et al. [2011], which was 
used here with age $=68$, height $=158 \mathrm{~cm}$ and weight $=113 \mathrm{Kg}$. The nonlinear part was taken to coincide with the generalized Hill equation (3) and the corresponding parameters $\gamma$ and $\mu$ were identified in Mendonça et al. [2012] from the surgery data, being given by: $\gamma=1.09$ and $\mu=2.40$.

In order to test the proposed control retuning strategy, the controller is first tuned assuming that the simulated patient is modeled by the parsimonious parameter Wiener model of Silva et al. [2010], with parameters $\alpha, \eta$, and $\gamma$ identified as in Mendonça et al. [2012]. These parameters were made available to us by the authors in a private communication and are given by $\alpha=0.0687, \eta=4.5413$, and $\gamma=1.09$. In order to increase the misfit between the theoretical model, PPM, and the simulated patient model, instead of the value $\mu=2.40$, identified for the patient, another value for $\mu$ is chosen in the PPM, namely $\mu=1.79$. This value is the average of the values for $\mu$ taken from a bank of identified values for eighteen real patients obtained in the work developed in Mendonça et al. [2012]

Figure 1 shows the BIS response of the patient using the controller (7) without applying the retuning strategy. In this case, the BIS response of the patient converged to 67 instead for the desired reference value, $z^{*}=50$, i.e., the BIS is affected by an error of $34 \%$.

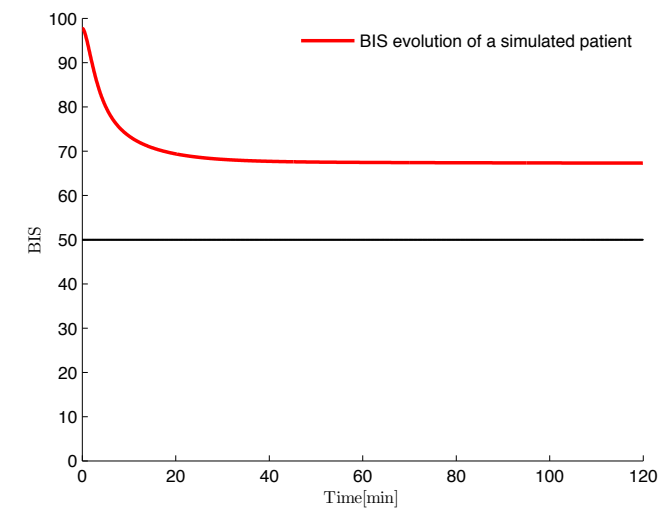

Fig. 1. BIS evolution of a simulated patient without applying the retuning strategy. The desired BIS level is set to be 50 .

The illustration of the BIS response of the patient with the retuning strategy is presented in Figures 2 and 3. In Fig. 2 the new reference $M^{*}$ was computed at time $t_{1}=20 \mathrm{~min}$ (when steady state was almost achieved), improving the BIS response of this patient, which converged to 48 , value quite close to the desired one. The error of $4 \%$, occurred in this case, would be smaller if the retunig happened latter in steady state. However, another retuning may be made, as shown in Fig. 3, where a second retuning occurred at the instant $t_{2}=60 \mathrm{~min}$ (when the steady state was achieved), which led the BIS response to converge to the desired value $z^{*}=50$.

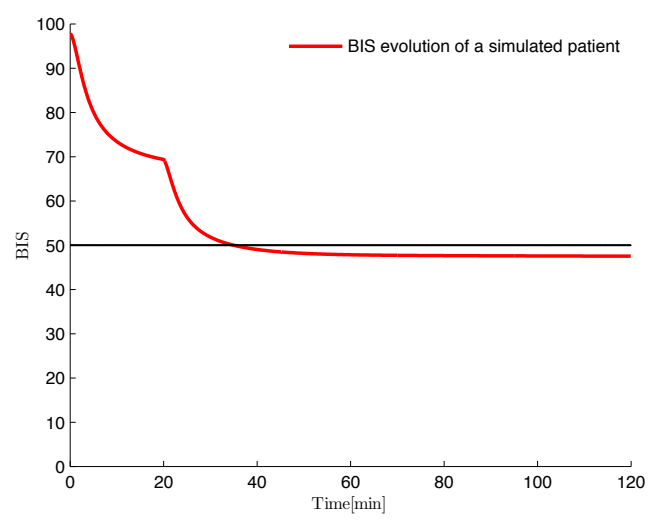

Fig. 2. BIS evolution of a patient modeled by a PK/PD model with a retune strategy applied at time $t=$ 20 min. The desired BIS level is set to be 50 .

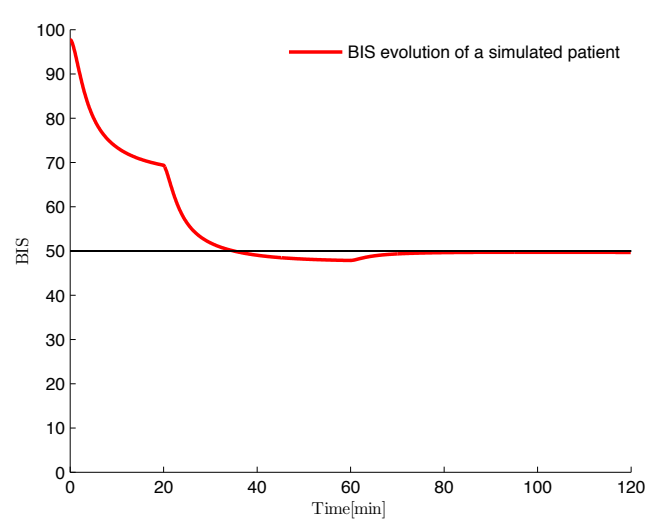

Fig. 3. BIS evolution of a patient modeled by a PK/PD model with a retune strategy applied at time instants $t_{1}=20 \mathrm{~min}$ and $t_{2}=60 \mathrm{~min}$. The desired BIS level is set to be 50 .

\section{EPILOGUE}

In this paper, a controller retuning strategy was proposed in order to improve the BIS reference tracking in patients, by means of simultaneous administration of propofol and of remifentanil, in the presence of model uncertainties. For this purpose, the patient is simulated by the PK/PD Wiener model proposed in Marsh et al. [1991], Minto et al. [1997], and Schnider et al. [1998], while the controller is tuned for a PPM. Assuming that the misfit in the patient response is due to the existence of errors in the parameters of the PPM, a controller retuning strategy is obtained that improves the reference tracking, as has been shown in the presented simulations.

\section{REFERENCES}

J. M. Bailey and W. M. Haddad. Drug dosing control in clinical pharmacology. Control Systems Magazine, IEEE, 25:35 - 51, 2005.

A. Ekman, M.L. Lindholm, C. Lennmarken, and R. Sandin. Reduction in the incidence of awareness using bis monitoring. Acta Anaesthesiol Scand, 48:20 $-26,2004$. 
R.J. Grindstaff and J.D. Tobias. Applications of bispectral index monitoring in the pediatric intensive care unit. $J$ Intensive Care Med, 19:111 - 116, 2004.

Clara M. Ionescu, Robin De Keyser, and Michel M.R.F. Struys. Evaluation of a propofol and remifentanil interaction model for predictive control of anesthesia induction. Proceedings of the 50th IEEE Conference on Decision and Control and European Control Conference (CDC-ECC), 2011.

B. Marsh, N. Norton, and G. N. Kenny. Pharmacokinetic model driven infusion of propofol in children. $\mathrm{Br} . \mathrm{J}$. Anaesthesia, 67:41 - 48, 1991.

T. Mendonça, H. Alonso, M. M. Silva, S. Esteves, and M. Seabra. Comparing different identification approaches for the depth of anesthesia using bis measurements. 16th IFAC Symposium on System Identification, 2012.

C. F. Minto, T. W. Schnider, T. D. Egan, E. Youngs, H. J. Lemmens, P. L. Gambus, V. Billard, J. F. Hoke, K. H. Moore, D. J. Hermann, K. T. Muir, J. W. Mandema, and S. L. Shafer. Influence of age and gender on the pharmacokinetics and pharmacodynamics of remifentanil. i. model development. Anesthesiology, $86: 10-23,1997$.

C. F. Minto, T. W. Schnider, T.G. Short, K.M. Gregg, A. Gentilini, and S. L. Shafer. Response surface model for anesthetic drug interactions. Anesthesiology, 92:1603 $-1616,2000$.

F. Nogueira, T. Mendonça, and P. Rocha. Output reference tracking for miso positive systems in general anesthesia. Proceedings of the 8th IFAC Symposium on Biological and Medical Systems, 8, 2012.

F. Nogueira, T. Mendonça, and P. Rocha. Improvement of the bis reference tracking performance in the presence of parameters uncertainties. Proceedings of the 21st Mediterranean Conference on Control and Automation - MED13, pages 219-224, 2013.

F. Nogueira, T. Mendonça, and P. Rocha. Controlling the depth of anesthesia by a novel positive control strategy. Computer Methods and Programs in Biomedicine - pii: S0169-2607(13)00406-9. doi: 10.1016/j.cmpb.2013.12.016., 2014.

T. W. Schnider, C. F. Minto, P. L. Gambus, C. Andresen, D. B. Goodale, S. L. Shafer, and E. J. Youngs. The influence of method of administration and covariates on the pharmacokinetics of propofol in adult volunteers. Anesthesiology, 8:1170 - 1182, 1998.

M. M. Silva, T. Mendonça, and T. Wigren. Online nonlinear identification of the effect of drugs in anaesthesia using a minimal parameterization and bis measurements. Proceedings of the American Control Conference (ACC10), pages 4379-4384, 2010.

C. Tirén, R.E. Anderson, G. Barr, A. Owall, and J.G. Jakobsson. Clinical comparison of three different anaesthetic depth monitors during cardiopulmonary bypass. Anaesthesia, 60:189 - 193, 2005.

S. D. Whyte and P. D. Booker. Monitoring depth of anaesthesia by eeg. British Journal of Anaesthesia, 3: $106-110,2003$.

E. Wodey, O. Tirel, J. Y. Bansard, A. Terrier, C. Chanavaz, R. Harris, C. Ecoffey, and L. Senhadji. Impact of age on both bis values and eeg bispectrum during anaesthesia with sevoflurane in children. $\mathrm{Br} J$ 